\title{
A quantum statistical theory of excitonic linewidth in semiconductor quantum well structures
}

\author{
K.K. BAJAJ and S.M. LEE* \\ Department of Physics, Emory University, Atlanta, GA 30322, U.S.A. \\ ${ }^{*}$ Department of Electrical Engineering, University of New Orleans, New Orleans, LA 70148, U.S.A.
}

\begin{abstract}
We have developed a very general and a powerful formalism to calculate the linewidths of excitonic transitions in semiconductor quantum well structures with arbitrary potential profiles in the presence of applied electric and magnetic fields. We assume that at low temperatures the dominant mechanism responsible for line broadening is the interface roughness which is always present even in the so called "perfect" samples. We have calculated the variation of the linewidth of the heavy-hole exciton $(\sigma)$ as a function of well size and magnetic and electric fields in $\mathrm{GaAs}-\mathrm{Al}_{0.3} \mathrm{Ga}_{0.7} \mathrm{As}$ based quantum well structures with three different potential well profiles, namely, rectangular, parabolic, and asymmetric triangular. We find that for a given value of the magnetic field the value of $\sigma$ increases as the well width is reduced, in all three different structures. For well sizes larger than about $100 \mathrm{~A}^{\circ}$, the value of $\sigma$ is the highest in the triangular wells and lowest in the rectangular wells. However, for well width less than $100 \mathrm{~A}^{\circ}$, the value of $\sigma$ falls below those in rectangular or parabolic wells. Also, for a given value of the well size the value of $\sigma$ increases with the magnetic field in all three different quantum well structures. It should be pointed out that our values of the excitonic linewidths are the lowest possible in these structures.
\end{abstract}

\section{INTRODUCTION}

Luminescence spectroscopy is one of the most powerful and useful techniques for obtaining information on the quality of semiconductor heterostructures such as quantum wells, and semiconductor alloys. The width of the excitonic transitions at low temperatures in quantum well structures provides an excellent measure of the quality of the interfaces and of the barrier alloy especially in the case of narrow wells, where a significant part of the wave function resides in the barrier. The excitonic transitions in unintentionally doped semiconductor alloys are generally broader than those observed in their components. This broadening has been attributed to the presence of compositional disorder in the alloys.

In this paper we present a calculation of the linewidth of excitonic transitions in semiconductor quantum well structures with arbitrary potential profiles in the presence of electric and magnetic fields. We assume that at low temperatures the dominant mechanism responsible for line broadening is the interface roughness which is always present even in the so called "perfect" structures. For instance, in the case of GaAs-AlGaAs quantum well structures, the AlGaAs layers at the interfaces have a completely random distribution of $\mathrm{Al}$ and $\mathrm{Ga}$ in the ideal material and will always lead to excitonic line broadening. We have generalized the formalism which we recently developed [1] to calculate the excitonic linewidths in semiconducto: alloys due to compositional disorder and have applied it to the calculation of the excitonic linewidths in quantun well structures. In particular, we have calculated the variation of the linewidth $(\sigma)$ of a heavy-hole exciton as a 


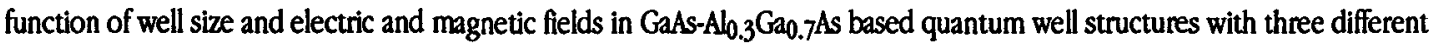
potential well profiles, namely, rectangular, parabolic and asymmetric triangular. Our formalism is general enough to be applicable to any quantum well structure where the quantum wells consist of binaries or alloys.

\section{FORMALISM}

In the following we briefly review the main features of our formalism as applied to the calculation of the excitonic linewidth in semiconductor alloys due to compositional disondering. We will then outline the generalization of this formalism for the calculation of excitonic linewidths in quantum well structures with arbitrary potential profiles in the presence of electric and magnetic fields.

A Wannier exciton in a semiconductor is described by its wave function which is determined by solving an appropriate Schrödinger wave equation. Since the exciton wave function, in principle, spreads throughout the semiconductor alloy, any definition of a so-called volume associated with the exciton becomes somewhat ambiguous. Thus the validity of the commonly used picture of an exciton subject to local potential fluctuations, whose range is much less than the exciton volume, becomes questionable [2-4].

We have developed a quantum statistical mechanical approach to calculate the excitonic line broadening in semiconductor alloys due to compositional disordering. We treat the exciton as a quantum mechanical system with a well defined wave function and therefore completely eliminate the need of using such ambiguous concepts as exciton volume. We calculate the exciton transition energy subject to the fluctuating band gap of any particular alloy configuration using first order perturbation theory. To obtain the excitonic lineshape, we sum over the contributions of all possible alloy configurations.

The exciton experiences a fluctuating potential due to the difference in bond strengths between alloy components, which is a rapidly varying function of spatial coordinates compared to the exciton wave function. Therefore, the excitonic properties are generally obtained using the average material parameters in this region. However, excitons generated in different places of the crystal experience different band gaps due to the random distributions of the alloy components. A detailed description of the fluctuating potential is clearly very complicated. We therefore estimate this fluctuating potential using a simple description, i.e., the virtual crystal approximation. There is either an atom of component $A$ or $B$ occupying each unit cell with a probability of $x$ or $(1-x)$, respectively. The difference in the bond strengths of $A$ and $B$ is the source of the fluctuating potential in the alloy. $E_{A}$ and $E_{B}$ are the band gaps of $\mathrm{A}$ and $\mathrm{B}$ components, respectively; $E_{g}$ is the average band gap, and $\Delta E_{g}$ is the fluctuating potential.

In a random binary alloy, the average energy band gap is given as

$$
E_{8}(x)=E_{A}+C_{A} x+C_{A B} x^{2}
$$

where $x$ is the concentration of atom $\mathrm{A}$ in the alloy, and $C_{\mathrm{AB}}$ is contribution from the lattice disorder generated by the random distributions of components $A$ and $B$ in the alloy. The radiative transition energy associated with the exciton ground state is defined as

$$
E_{\text {trans }}(\mathbf{r})=E_{g}(\mathbf{r})+\frac{1}{2} \hbar \omega_{c}-E_{b}
$$

where $E_{b}$ is the exciton binding energy, and $\omega_{c}$ is the cyclotron frequency. One must keep in mind that Eq. (1) describes the average band gap. On a microscopic scale, however, there is either an atom of component A or B occupying each unit cell with a probability of $x$ or $(1-x)$, respectively. Using the virtual crystal approximation, we approximate the fluctuating potential as the band-gap variation from its average value at the unit cell about the point $\mathbf{r}=\mathbf{r}_{\mathrm{ijk}}$ as

$$
\Delta E_{g}\left(s_{i j k}\right)=E_{g}\left(s_{i j k}\right)-E_{g}(x)
$$

where $s_{i j k}$ is 0 for $A$ or 1 for $B$ residing at the site near $\mathbf{r}_{i j k}$.

When an exciton is present in the alloy, it experiences fluctuating potentials from many unit cells. Although these local fluctuations are very large, they tend to cancel each other out. The excitonic transition energy is much larger than its linewidth. Therefore, we use a first-order perturbation theory for the excitonic transition energy in the presence of the fluctuating potentials, which should be accurate in calculating the line-shape function, 


$$
\begin{aligned}
E & =E_{g}(x)-E_{b}+\int \Delta E_{z}(\mathbf{r})|\psi(\mathbf{r})|^{2} d \mathbf{r} \\
& =E_{z}(x)-E_{b}+\sum_{i j k} \Delta E_{g}\left(s_{i j k}\right)\left|\psi_{i j k}\right|^{2} \Delta V,
\end{aligned}
$$

where $\Delta V=a^{3} / 4$ is the unit cell volume for a zincblende structure with $a$ the lattice constant of the binary alloy and $\psi(r)$ is the excitonic wave function.. The last term in Eg. (4) sums over all unit cells in the crystal.

The details of the calculations of the values of the excitonic transition energies due to different alloy configurations and hence the line shape function are given in Ref. 1.

We now discuss the application of this formalism to calculate the excitonic linewidth in quantum well structures. In a rectangular $\mathrm{GaAs} / \mathrm{Al}_{0.3} \mathrm{Ga}_{0.7} \mathrm{As}$ quantum well structure, the effects of the broadening arise from the compositional disorder present at the interfaces and in the barrier alloy. We assume abrupt interfaces and calculate the line broadening using exciton wave functions appropriate for this quantum well structure. In graded quantum well structures such as parabolic and asymmetric triangular, we calculate the exciton linewidth by reganding them as inhomogenous alloys. As pointed out earlier, in our formalism we sum over the contributions from all possible alloy configurations and therefore the extension of our formalism to quantum well structures with arbitrary potential profiles is quite straightforward.

\section{RESULTS AND DISCUSSION}

In Fig. 1, we display the variation of the linewidth $(\sigma)$ of a heavy-hole exciton as a function of well size in square, parabolic and asymmetric triangular $\mathrm{CaAs} / \mathrm{Al}_{03} \mathrm{Ga}_{0.7} \mathrm{As}$ quantum well structures. We find that the value of $\sigma$ increases as the well size is reduced, in all three different structures, as expected. For well sizes larger than $100 \mathrm{~A}^{\circ}$, the value of $\sigma$ is the highest in the asymmetric triangular wells and lowest in the rectangular wells. However, for well widths less than $100 \mathrm{~A}^{\circ}$, the value of $\sigma$ in asymmetric triangular wells falls below those in rectangular or parabolic wells. This may be due to the fact that in narrow asymmetric triangular quantum wells the excitonic linewidth corresponds to an average alloy composition which is much less than 0.3 whereas in narrow rectangular wells much of the excitonic wave function resides in the barriers thus experiencing the effect of higher alloy composition.

In Fig. 2, we display the variation of $\sigma$ as a function of well size in rectangular quantum wells for several values of electric and magnetic fields applied parallel to the direction of growth. We find that for a given well size, the value of $\sigma$ increases as a function of the magnetic field due to the shrinkage of the excitonic wave function. Also for a given value of the well size and magnetic field the value of $\sigma$ increases as a function of the applied electric field. This is due to the fact that the application of the electric field pushes the excitonic wave function into the barrier. Similar results are also obtained for parabolic and asymmeuric triangular quantum wells, and for light-hole excitons.

\section{REFERENCES}

*Work supported by the Air Force Office of Scientific Research under Grant No: AFOSR-90-0118.

1. Lee S. M. and Bajaj K. K., J. Appl. Phys. 73 (1993) 1788 - 1790

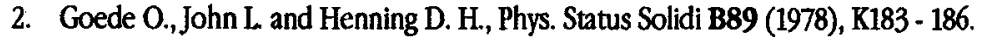

3. Singh J. and Bajaj K. K., Appl. Phys. Lett. 44 (1984) 1075 - 1077.

4. Schubert E. F., Gobel E, Horikoshi O., Plough Y. K. and Queisser, H. J., Phys. Rev. B 30 (1984) 813 - 820. 


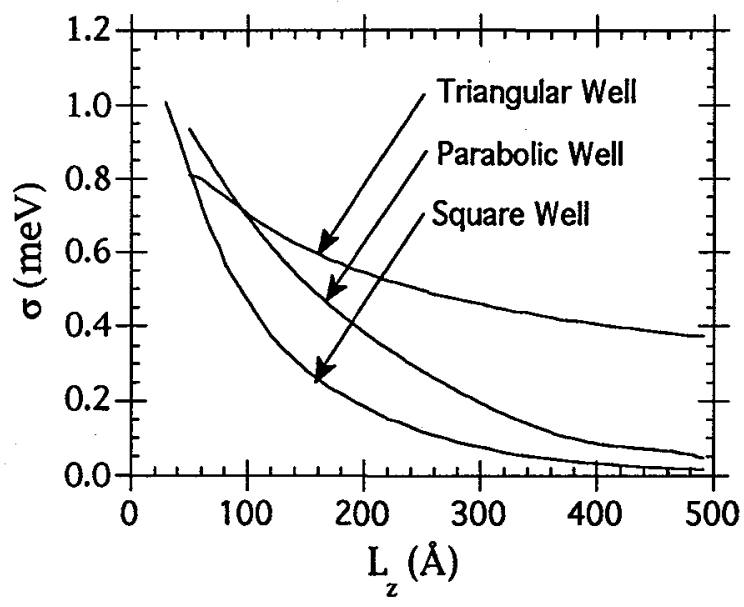

Fig. 1 Variation of the linewidth $(\sigma)$ of a heavy-hole exciton as a function of well size in a square, parabolic and asymmetric triangular $\mathrm{GaAs} / \mathrm{Al}_{0.3} \mathrm{Ga} 0.7$ As quantum well structures.

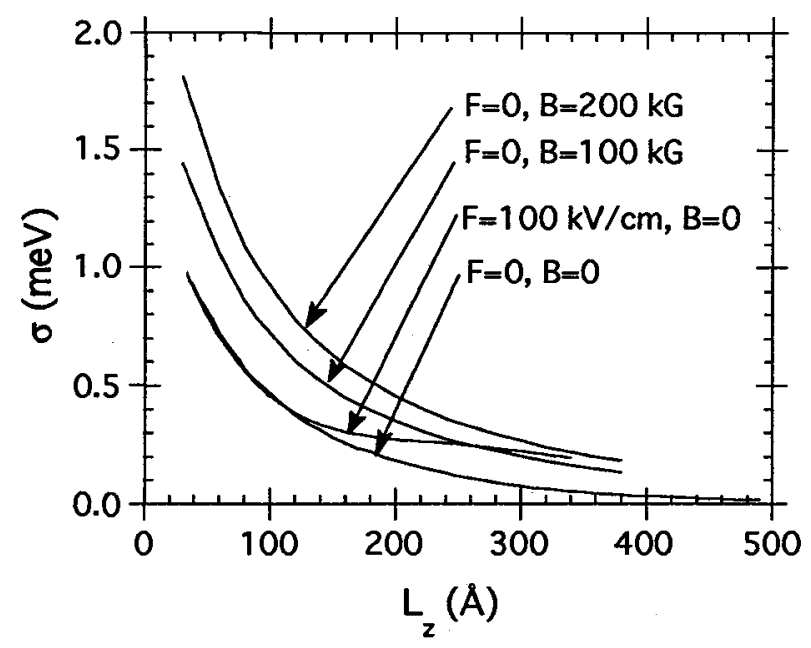

Fig. 2 Variation of the linewidth $(\sigma)$ of a heavy-hole exciton as a function of well size (Lz) in a square $\mathrm{GaAs}-\mathrm{Al}_{0.3} \mathrm{Ga}_{0.7} \mathrm{As}$ quatnum well for several values of the electric ( $\mathrm{F}$ ) and magnetic (B) fields. 